\title{
Iterative Decoding Convergence and Termination of Serially Concatenated Codes
}

\author{
Robert G. Maunder, Member, IEEE, and Lajos Hanzo, Fellow, IEEE
}

\begin{abstract}
In this paper, we demonstrate that the previously proposed arrangements of serially concatenated codes have extrinsic-information-transfer (EXIT) functions that intersect each other at points that are near but not at the $(1,1)$ point in the top-right-hand corner of the EXIT chart, which is typically associated with elevated error floors. We propose a novel arrangement having EXIT functions that do not intersect before the $(1,1)$ point, which is typically associated with approaching the maximumlikelihood (ML) bit-error-ratio (BER) performance. Our method employs an inner recursive code that is terminated using specifically designed termination sequences, which have a minimum Hamming distance of at least two between each other. Additionally, we provide optimal termination sequences for a range of inner code designs. Finally, we demonstrate that our novel approach can facilitate useful BER reductions in the challenging application scenario when employing short frame lengths on the order of 100 bits, which are typical in wireless sensor networks, for example.
\end{abstract}

Index Terms-Convolutional codes, error-correction coding, information rates, trellis codes.

\section{INTRODUCTION}

$\mathbf{E}$ XTRINSIC-information-transfer (EXIT) charts [1] have been shown to characterize the iterative exchange of extrinsic information between serially concatenated decoders [2], [3]. This is illustrated in the schematic in Fig. 1(a), where $\tilde{\mathbf{b}}_{\mathrm{e}}$ and $\tilde{\mathbf{c}}_{\mathrm{e}}$ are the extrinsic logarithmic-likelihood-ratio (LLR) sequences exchanged. An EXIT chart is said to have a closed tunnel when the EXIT functions of the inner and outer decoders intersect each other, as exemplified in Fig. 2(a). A relatively high bit error ratio (BER) will typically result in this case, because the iterative decoding trajectory [1] is prevented from converging toward the top-right-hand corner of the EXIT chart, which is the only point where maximum-likelihood (ML) decoding is achieved.

If both serially concatenated decoders are capable of providing extrinsic LLRs $\left(\tilde{\mathbf{b}}_{\mathrm{e}}\right.$ or $\tilde{\mathbf{c}}_{\mathrm{e}}$ ) having the maximal mutual information $\left[I\left(\tilde{\mathbf{b}}_{\mathrm{e}} ; \mathbf{b}\right)\right.$ or $\left.I\left(\tilde{\mathbf{c}}_{\mathrm{e}} ; \mathbf{c}\right)\right]$ of unity [1] when presented with a priori LLRs $\left(\tilde{\mathbf{b}}_{\mathrm{a}}\right.$ or $\left.\tilde{\mathbf{c}}_{\mathrm{a}}\right)$ that have unity mutual information $\left[I\left(\tilde{\mathbf{b}}_{\mathrm{a}} ; \mathbf{b}\right)\right.$ or $\left.I\left(\tilde{\mathbf{c}}_{\mathrm{a}} ; \mathbf{c}\right)\right]$, then their EXIT functions will both reach the $\left[I\left(\tilde{\mathbf{b}}_{\mathrm{e}} ; \mathbf{b}\right)\right.$ or $\left.I\left(\tilde{\mathbf{c}}_{\mathrm{e}} ; \mathbf{c}\right)\right]=(1,1)$ point at the topright-hand corner of the EXIT chart. In this case, an open EXIT chart tunnel can be created for sufficiently high channel signal to noise ratios (SNRs) $E_{c} / N_{0}$, as exemplified in Fig. 2(c). Provided that the EXIT functions can adequately predict the path of

Manuscript received July 9, 2008; revised July 9, 2009. First published July 21, 2009; current version published January 20, 2010. The review of this paper was coordinated by G. Bauch.

The authors are with the School of Electronics and Computer Science, University of Southampton, SO17 1BJ Hampshire, U.K. (e-mail: rm@ecs.soton. ac.uk.; 1h@ecs.soton.ac.uk).

Digital Object Identifier 10.1109/TVT.2009.2028118 the iterative decoding trajectory, this will then converge toward the $(1,1)$ point, as the iterative decoding process proceeds. Meanwhile, the resultant BER will asymptotically approach the ML performance but at a fraction of the ML decoder's complexity [4].

Note that the ML BER performance depends upon the spectrum of Hamming distances [5] between the possible permutations of the transmitted bit sequence, which is labeled $\mathbf{d}$ in Fig. 1(a). Previously, efforts like those in [5] and [6] have been made to characterize the distance spectrum of serially concatenated codes to assist the design of improved iteratively decoded schemes. However, these efforts have limited benefit if the iterative decoder's BER performance is prevented from approaching that of the ML decoder. For this reason, it is critical that both of the concatenated decoders have EXIT functions that reach the $(1,1)$ point of the EXIT chart when the lowest possible BERs are sought.

Recently, a flurry of serial concatenations employing recursive convolutional inner codes have been proposed, including those in [6]-[15], for example. While these schemes can have EXIT functions that reach the $(1,1)$ point of the EXIT chart, this is only achieved in the impractical case where the recursive code has an infinite length, as detailed in [16] and [17]. For long but finite code lengths, the EXIT functions will intersect each other at near- $(1,1)$ points, although this distinction is often avoided in the corresponding publications. Furthermore, the intersection point can significantly be shifted away from the $(1,1)$ point if short codes are employed, as will be demonstrated in Section IV. In these cases, relatively high BERs can result, as will be shown in Section V. This is of particular concern when employing an irregular inner code [15], for example, since these typically use short component codes.

This discussion motivates the novel contribution of this paper. More specifically, in Section II, we propose a scheme that has EXIT functions that do not intersect until the $(1,1)$ point of the EXIT chart, despite employing a recursive inner code having a finite length. We believe this to be the first time that this has been demonstrated for the type of scheme considered. This feat is achieved by terminating the recursive inner code using bit sequences that have a minimum Hamming distance $d_{\mathrm{f}}$ of at least two between each other [18]. This is in contrast with the approaches in [6], [9]-[11], [19], and [20], which employ termination sequences that have a minimum Hamming distance of $d_{\mathrm{f}}=1$ between each other. These schemes therefore have EXIT functions that intersect each other, as we shall show in Section IV.

Similar to the termination of parallel concatenations of recursive codes [21], our novel approach imposes some restrictions 


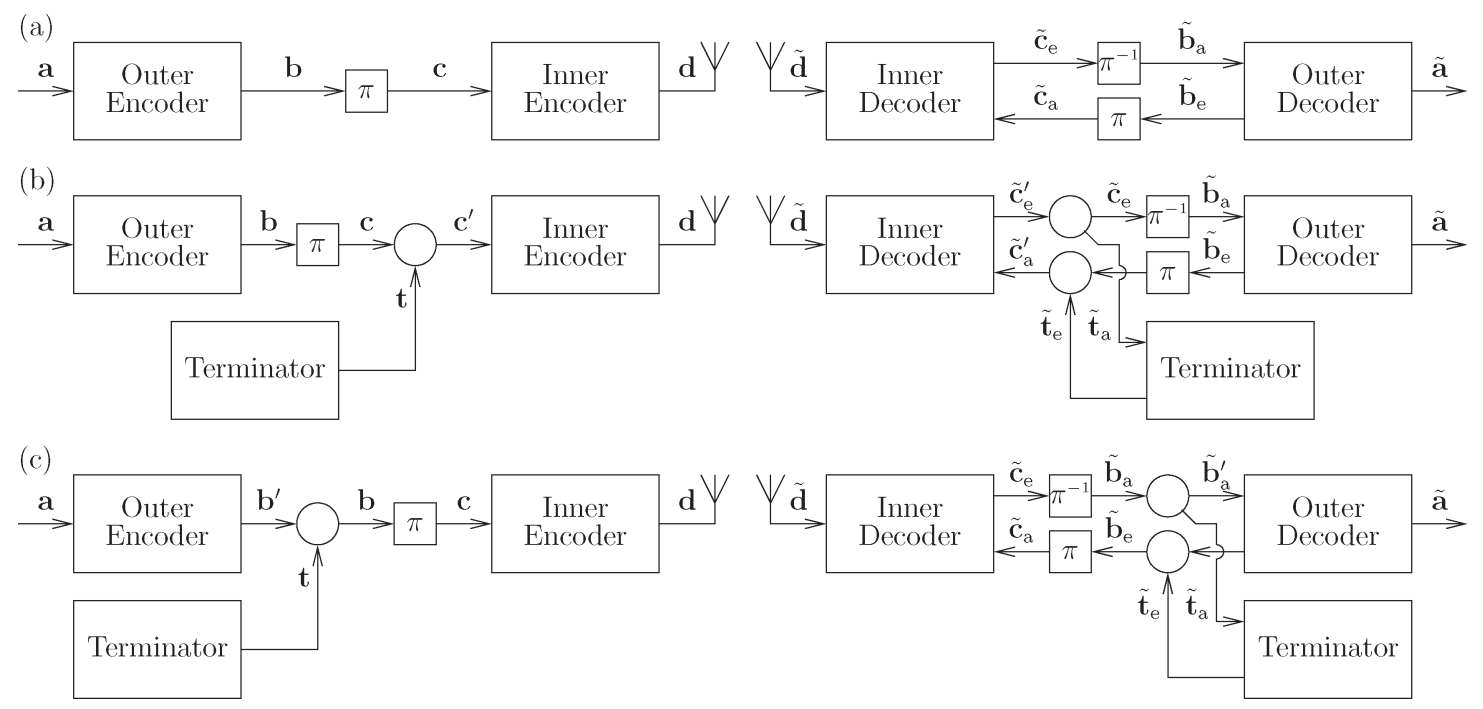

Fig. 1. Schematics of various serially concatenated code arrangements. The various bit sequences employed in the transmitters are labeled. The corresponding LLR sequences employed in the receivers are indicated using tildes. A subscript of "a" indicates a priori information, while a subscript of "e" is employed for extrinsic information. The circular junctions indicate either a multiplexing or demultiplexing operation.

upon the design of both the termination sequences and the interleaver. These restrictions are discussed in Section III. Note that, while the problem of code termination in parallel concatenations received a significant amount of attention [21]-[25] more than a decade ago, termination in serial concatenations has remained neglected until now. This may be attributed to the additional challenges that are faced when terminating serially concatenated codes. More specifically, parallel concatenations can be terminated by manipulating the uncoded bit sequence [21], since this (or an interleaved version of it) directly feeds into all concatenated codes. By contrast, in a serial concatenation, the uncoded bit sequence is encoded by the outer encoder, before the inner encoder is invoked. As a result, manipulation of the uncoded bit sequence cannot be employed to directly terminate the inner code.

After examining the EXIT characteristics of our proposed scheme and comparing them with those of suitably designed benchmarkers in Section IV, we shall compare the BER performance of these schemes in Section V. Finally, we will offer our conclusions in Section VI.

\section{TRELLIS TERMINATION}

In the scheme in Fig. 1(a), the serially concatenated decoders generate extrinsic LLRs $\tilde{\mathbf{x}}_{\mathrm{e}}$ pertaining to the bits in the sequence $\mathrm{x}$ by considering the a priori LLRs $\tilde{\mathbf{x}}_{\mathrm{a}}$ provided by the other decoder, where $\mathbf{x} \equiv \mathbf{b}$ in the case of the outer decoder, and $\mathbf{x} \equiv$ $\mathbf{c}$ in the case of the inner decoder. These LLR sequences will have mutual informations of unity if they identify the values of the corresponding bits in $\mathrm{x}$ with absolute confidence [1]. If the particular decoder generates an extrinsic mutual information of $I\left(\tilde{\mathbf{x}}_{\mathrm{e}} ; \mathbf{x}\right)=1$ whenever the a priori mutual information is $I\left(\mathbf{x}_{\mathrm{a}} ; \mathbf{x}\right)=1$, then the corresponding EXIT function will reach the $(1,1)$ point of the EXIT chart. This will be achieved if the decoder is able to infer the value of each bit in $\mathrm{x}$ with absolute certainty, given the unequivocal a priori knowledge of all other bit values in $\mathrm{x}$. This is because the decoders generate the extrinsic information $\tilde{\mathbf{x}}_{\mathrm{e}}$ pertaining to each particular bit in the corresponding sequence $\mathrm{x}$ by considering the a priori information $\tilde{\mathbf{x}}_{\mathrm{a}}$ provided for each of the other bits in $\mathbf{x}$ [4].

Let us begin by considering the outer code in Fig. 1(a). Suppose that there was a minimum Hamming distance of one between the legitimate permutations of the bit sequence $b$ generated by the outer encoder. Here, a single unknown bit value could be the only difference between the correct permutation of $\mathbf{b}$ and another. In this case, the decoder would not be able to decide which of these two permutations was the correct one. Hence, the unknown bit value would not be determined, even though the decoder has unequivocal knowledge of all other bit values. As a result, the outer code's EXIT function would not reach the $(1,1)$ point of the EXIT chart, as previously described. By contrast, if the outer code imposed a minimum Hamming distance of at least two, then a single unknown bit value could never be the only difference between the correct permutation and another. In this case, the decoder can always identify the correct permutation without ambiguity, and the value of the missing bit will be determined with absolute certainty. Hence, imposing a minimum Hamming distance of at least two is a necessary and sufficient condition for the outer code's EXIT function to reach the $(1,1)$ point of the EXIT chart, as was formally proven in [18]. This criterion is satisfied by a repetition code, for example.

Similarly, for the inner decoder to have an EXIT function that reaches the $(1,1)$ point, it must be able to infer the value of any single uncoded bit value in $\mathbf{c}$ with absolute certainty, given perfect a priori knowledge of all other bit values in c, even if the amount of information available for the coded bits of $\mathbf{d}$ is infinitesimally low. A sufficient condition for this is satisfied when the inner code is a recursive convolutional code (such as a rate-1 code [7]) having an infinite length, as detailed in [16] and [17] and alluded to in Section I. However, this condition is not satisfied when the recursive convolutional code has a finite length [16], [17]. In common with all other types of convolutional encoders [26], a recursive encoder employs $m$ 
(a)

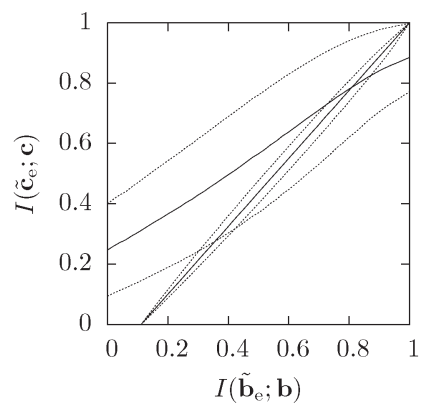

(b)

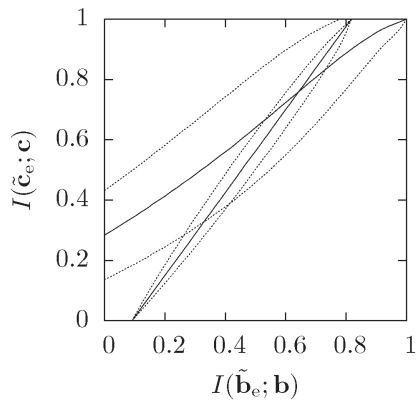

(c)

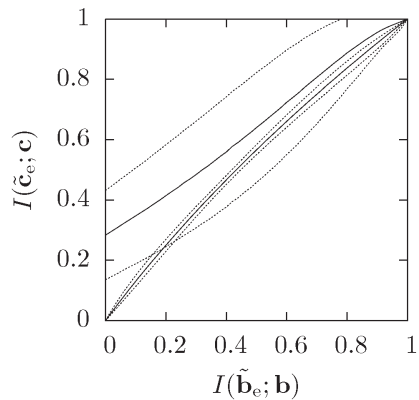

(d)

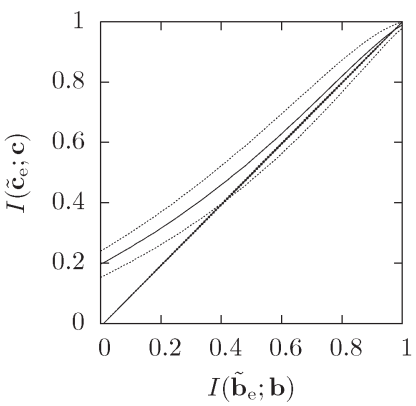

(e)

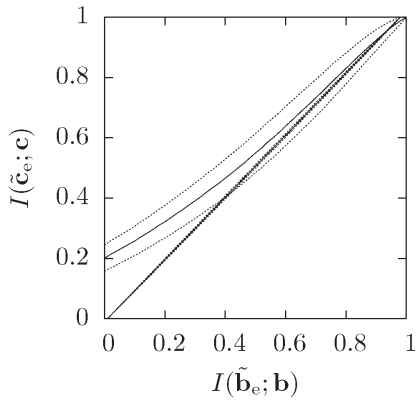

(f)

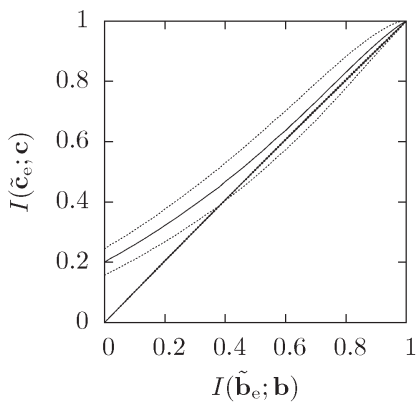

Fig. 2. EXIT band charts of the schemes introduced in Section II, when transmitting over a BPSK-modulated uncorrelated narrow-band Rayleigh fading channel at an SNR of $E_{c} / N_{0}=0 \mathrm{~dB}$. The dotted lines represent the EXIT bands, which surround the EXIT functions that are plotted using continuous lines. (a) No termination, $n_{\mathbf{a}}=4$, and $n_{\mathbf{d}}=9$. (b) $d_{\mathrm{f}}=1$ termination, $n_{\mathbf{a}}=$ 4 , and $n_{\mathbf{d}}=9$. (c) $d_{\mathrm{f}} \geq 2$ termination, $n_{\mathbf{a}}=4$, and $n_{\mathbf{d}}=9$. (d) No termination, $n_{\mathbf{a}}=49$, and $n_{\mathbf{d}}=99$. (e) $d_{\mathrm{f}}=1$ termination, $n_{\mathbf{a}}=49$, and $n_{\mathbf{d}}=$ 99. (f) $d_{\mathrm{f}} \geq 2$ termination, $n_{\mathbf{a}}=49$, and $n_{\mathbf{d}}=99$.

binary memory elements. Each bit of the encoded sequence $\mathbf{d}$ is generated as a function of both the corresponding bit in the input bit sequence $\mathbf{c}$ and the contents or state of the encoder's memory. However, in contrast to nonrecursive encoders, the state of a recursive encoder depends upon the values of the previous bits in the encoded sequence $\mathbf{d}$, as well as in the uncoded sequence $\mathbf{c}$. Encoding typically commences from a particular state that is known to the recursive decoder. However, when a recursive encoder finishes encoding a bit sequence $\mathbf{c}$ having a finite length, it will enter a particular final state that is not inherently known to the decoder. It is the decoder's uncertainty about the final state that prevents it from inferring the value of any single uncoded bit value in $\mathbf{c}$ with absolute certainty, even when provided with perfect $a$ priori knowledge of all other bits in c. As a result, the inner decoder in the scheme in Fig. 1(a) will have an EXIT function that does not reach the $(1,1)$ point of the EXIT chart when $\mathbf{c}$ has a finite length, as was formally proven in [16] and [17]. This is exemplified in Fig. 2(a) and discussed in Section IV, where this scheme is referred to as employing "no termination."

However, code termination [27] can be employed to force the inner encoder into a particular final state that is expected by the decoder. As shown in Fig. 1(b), an uncoded bit sequence $\mathbf{c}^{\prime}$ that achieves this may be obtained by considering the uncoded bit frame $\mathbf{c}$ and appending a sequence of specially selected terminating bits $\mathbf{t}$. Note that attaching a terminating sequence $\mathbf{t}$ comprising $m$ bits is always sufficient to terminate the inner encoder in the desired final state [27].

With this arrangement, the inner decoder will be aware of both the initial and final encoder states. It can therefore employ a trellis that is terminated at both ends to consider all possible reconstructions of the bit sequence $\mathbf{c}^{\prime}$ [27]. In this case, knowledge about all but one of the uncoded bits in $\mathbf{c}^{\prime}$ permits the decoder to recurse from each end of the trellis and identify the state entered before and after the remaining unknown bit, revealing its value. ${ }^{1}$ Hence, termination allows the inner decoder to obtain extrinsic LLRs $\tilde{\mathbf{c}}_{\mathrm{e}}^{\prime}$ having the maximal mutual information of $I\left(\tilde{\mathbf{c}}_{\mathrm{e}}^{\prime} ; \tilde{\mathbf{c}}^{\prime}\right)=1$ when provided with perfect a priori LLRs $\tilde{\mathbf{c}}_{\mathrm{a}}^{\prime}$ that have a mutual information of $I\left(\tilde{\mathbf{c}}_{\mathrm{a}}^{\prime} ; \tilde{\mathbf{c}}^{\prime}\right)=1$.

However, in Fig. 1(b), it is $\tilde{\mathbf{c}}_{\mathrm{e}}$ rather than $\tilde{\mathbf{c}}_{\mathrm{e}}^{\prime}$ that is forwarded to the outer decoder during iterative decoding. Therefore, to support iterative decoding convergence toward the $(1,1)$ point, we require the extrinsic LLRs of $\tilde{\mathbf{c}}_{\mathrm{e}}$ to have a mutual information of $I\left(\tilde{\mathbf{c}}_{\mathrm{e}} ; \mathbf{c}\right)=1$ when the a priori LLRs of $\tilde{\mathbf{c}}_{\mathrm{a}}$ have unity mutual information $I\left(\tilde{\mathbf{c}}_{a} ; \mathbf{c}\right)$. During iterative decoding, we obtain $\tilde{\mathbf{c}}_{\mathrm{e}}$ by removing $\tilde{\mathbf{t}}_{\mathrm{a}}$ from $\tilde{\mathbf{c}}_{\mathrm{e}}^{\prime}$ and, following outer decoding and interleaving, obtain $\tilde{\mathbf{c}}_{\mathrm{a}}^{\prime}$ by appending $\tilde{\mathbf{t}}_{\mathrm{e}}$ to $\tilde{\mathbf{c}}_{\mathrm{a}}^{\prime}$. However, unless we have a source of information for $\mathbf{t}_{\mathrm{e}}$, we must populate it using zero-valued LLRs, which represent the absence of information. In this case, the a priori LLRs $\tilde{\mathbf{c}} \mathrm{a}^{\prime}$ will never have unity mutual information $I\left(\tilde{\mathbf{c}}_{\mathrm{a}}^{\prime}, \mathbf{c}^{\prime}\right)$, even if $\tilde{\mathbf{c}}_{\mathrm{a}}$ does. As a result, neither $\tilde{\mathbf{c}}_{\mathrm{e}}^{\prime}$ nor $\tilde{\mathbf{c}}_{\mathrm{e}}$ will have unity mutual information, and hence, the scheme in Fig. 1(b) will have EXIT functions that intersect each other before the $(1,1)$ point of the EXIT chart. This is exemplified in Fig. 2(b) and discussed in Section IV, where this scheme is referred to as employing " $d_{\mathrm{f}}=1$ termination." Note that this is the termination method employed in [6], [9]-[11], [19], and [20], as described in Section I.

The described problem can be overcome by employing a code terminator that is capable of providing extrinsic LLRs $\tilde{\mathbf{t}}_{\mathrm{e}}$ that have the maximal mutual information of $I\left(\tilde{\mathbf{t}}_{\mathrm{e}} ; \mathbf{t}\right)=1$. This can be achieved by employing termination sequences $\mathbf{t}$ from a

\footnotetext{
${ }^{1}$ An alternative interpretation is that because the particular termination sequence $\mathbf{t}$ that is required depends upon the bit values of the uncoded sequence $\mathbf{c}$, the possible permutations of the resultant concatenation $\mathbf{c}^{\prime}$ will have a minimum Hamming distance of at least two between each other. As a result, the inner decoder becomes capable of inferring the value of a single missing bit in $\mathbf{c}^{\prime}$ in the same way that the outer decoder can, as previously described. Note that tailbiting [28] has been proposed as an alternative to termination for improving a recursive decoder's performance, since it does not impose the overhead of requiring termination bits. However, because of this, there is a minimum Hamming distance of unity between the possible permutations of the input to a recursive tailbiting encoder. As a result, the EXIT function of a recursive tailbiting decoder will not reach the $(1,1)$ point of the EXIT chart, as previously described.
} 
set that exhibit a minimum Hamming distance $d_{\mathrm{f}}$ of at least two between each other. This permits the terminator in Fig. 1(b) to generate extrinsic LLRs $\tilde{\mathbf{t}}_{\mathrm{e}}$ having a mutual information of $I\left(\tilde{\mathbf{t}}_{\mathrm{e}} ; \mathbf{t}\right)=1$ when provided with a priori LLRs $\tilde{\mathbf{t}}_{\mathrm{a}}$ that have a mutual information of $I\left(\tilde{\mathbf{t}}_{\mathrm{a}} ; \mathbf{t}\right)=1$, like the outer decoder, as previously described. Note that the termination sequences of $\mathbf{t}$ must comprise more than $m$ bits for the minimum Hamming distance $d_{\mathrm{f}}$ between them to be at least two. Furthermore, not all sets of bit sequences having a minimum Hamming distance of at least two between each other are capable of terminating the inner code. Hence, in Section III, we shall provide optimal termination sequences for recursive inner codes having various feedback polynomials.

Observe in Fig. 1(b) that the bits of the termination sequence $\mathbf{t}$ remain close to each other within $\mathbf{c}^{\prime}$, since they are merely appended to $\mathbf{c}$. This is undesirable, because the inner decoder and the terminator assume the independence of all neighboring LLRs within $\tilde{\mathbf{c}}_{\mathrm{a}}^{\prime}$ and $\tilde{\mathbf{t}}_{\mathrm{a}}$, respectively [29]. Hence, this assumption is not satisfied because these LLR sequences are provided by $\tilde{\mathbf{t}}_{\mathrm{e}}$ and $\tilde{\mathbf{c}}_{\mathrm{e}}^{\prime}$, in which all neighboring LLRs are generated together by the terminator and the inner decoder, respectively.

The solution of this problem is to introduce the termination sequence $\mathbf{t}$ before interleaving takes place in block $\pi$ in Fig. 1(c). As a result, the bits within $\mathrm{t}$ do not remain close to each other within $\mathbf{c}$. Furthermore, this approach grants us some freedom when positioning the bits of $\mathbf{t}$ within $\mathbf{c}$, potentially facilitating a better distance spectrum and ML BER performance [6] than that attained by the scheme in Fig. 1(b). However, we are not granted absolute freedom when designing the interleaver $\pi$. More specifically, not all positionings of the bits of $\mathbf{t}$ within $\mathbf{c}$ are capable of terminating the inner code. We shall discuss the constraints governing the interleaver design in Section III. Note though that these constraints do not limit the positioning of the bits of $\mathbf{b}^{\prime}$ within $\mathbf{c}$. The EXIT characteristics of the scheme depicted in Fig. 1(c) will be investigated in Section IV, where the corresponding procedure is referred to as " $d_{\mathrm{f}} \geq 2$ termination."

\section{Terminator AND INTERLEAVER DESIGN}

Let us assume (without loss of generality) that the recursive inner encoder in Fig. 1(a) commences the encoding of the $n_{\mathbf{c}}$-bit sequence $\mathbf{c} \in\{0,1\}^{n_{\mathbf{c}}}$ in the "all-zero" state, where all $m$ of its binary memory elements store zero-valued bits. If $\mathbf{c}$ contains only a single logical one-valued bit (located at position $\left.i \in\left[1, \ldots, n_{\mathbf{c}}\right]\right)$, then the inner encoder will remain in the allzero state until this single "one" is encountered. After this point, the recursive inner encoder will emerge from and never return to the all-zero state, owing to its infinite impulse response [7]. When the encoding of the remaining $\left(n_{\mathbf{c}}-i\right)$ zero-valued bits in $\mathbf{c}$ is completed, the inner encoder will reside in a particular final state $\mathbf{s}_{i} \in\{0,1\}^{m}$, which depends upon the position $i$ of the one-valued bit within $\mathbf{c}$.

Note that the set of all these so-called impulse response final states $\left\{\mathbf{s}_{i}\right\}_{i=1}^{n_{\mathrm{c}}}$ will not include the all-zero state. Furthermore, owing to the regular structure of the trellis, $\left\{\mathbf{s}_{i}\right\}_{i=1}^{n_{\mathrm{c}}}$ will be periodic in $i$, having a period of less than $2^{m}$. In each period, no impulse response final states will appear more than once. Since
TABLE I

Optimal Terminator Generator Matrices G For VARious INNER-CODE FEEDBACK POLYNOMIALS $\mathbf{f}$

\begin{tabular}{|c|c|c|c|c|}
\hline f & G & $d_{\mathrm{f}}$ & $a_{\mathrm{f}}$ & $r$ \\
\hline$\left[\begin{array}{ll}1 & 1\end{array}\right]$ & {$\left[\begin{array}{lll}1 & 1 & 1\end{array}\right]$} & 3 & 1 & 1 \\
\hline$\left[\begin{array}{lll}1 & 1 & 1\end{array}\right]$ & {$\left[\begin{array}{lll}0 & 1 & 1 \\
1 & 0 & 1\end{array}\right]$} & 2 & 1 & 0.222 \\
\hline$\left[\begin{array}{lll}1 & 0 & 1\end{array}\right]$ & {$\left[\begin{array}{llll}0 & 1 & 1 & 1 \\
1 & 0 & 0 & 1\end{array}\right]$} & 2 & 0.333 & 0.5 \\
\hline$\left[\begin{array}{llll}1 & 0 & 1 & 1\end{array}\right]$ & \multirow{2}{*}[\begin{array}{llll}{0}&{0}&{1}&{1}\\
{0}&{1}&{1}&{0}\\
{1}&{0}&{0}&{1}\end{array}]{} & 2 & 0.857 & 0.28 \\
\hline$\left[\begin{array}{llll}1 & 1 & 0 & 1\end{array}\right]$ & & 2 & 0.857 & 0.28 \\
\hline$\left[\begin{array}{llll}1 & 0 & 0 & 1\end{array}\right]$ & \multirow{2}{*}[\begin{array}{lllll}{0}&{0}&{1}&{1}&{1}\\
{0}&{1}&{0}&{1}&{0}\\
{1}&{0}&{0}&{0}&{1}\end{array}]{} & 2 & 0.286 & 0.296 \\
\hline$\left[\begin{array}{llll}1 & 1 & 1 & 1\end{array}\right]$ & & 2 & 0.286 & 0.375 \\
\hline
\end{tabular}

recursive encoders are linear [21], the final state $\mathbf{s} \in\{0,1\}^{m}$ entered into when encoding an arbitrary $\mathbf{c}=\left\{c_{i}\right\}_{i=1}^{n_{\mathrm{c}}}$ can be determined using a sum over $\mathrm{GF}(2)$ according to

$$
\mathbf{s}=\sum_{i=1}^{n_{c}} c_{i} \mathbf{s}_{i} .
$$

Let us now consider the conditions required for the terminator and interleaver in Fig. 1(c) to be capable of terminating the inner encoder. These conditions will be satisfied if the various possible permutations of $\mathbf{t}$ allow the inner encoder to end up in any particular state $\mathbf{s} \in\{0,1\}^{m}$ after encoding $\mathbf{c}$. If this is the case, then the terminator can select the particular permutation of $\mathbf{t}$ that yields the specific final state $\mathbf{s}$ expected by the receiver. Since there are $2^{m}$ possible final states in the set $\{0,1\}^{m}$, we require $2^{m}$ termination sequences.

Our termination sequences can be represented by a $\left(2^{m} \times\right.$ $n_{\mathbf{t}}$ )-element matrix $\mathbf{T}=\left[\begin{array}{llll}\mathbf{t}_{1} & \mathbf{t}_{2} & \ldots & \mathbf{t}_{2^{m}}\end{array}\right]^{\mathrm{T}}$, where each termination sequence column vector $\mathbf{t}_{p} \in\{0,1\}^{n_{\mathbf{t}}}$ comprises $n_{\mathbf{t}}>m$ bits, where $p \in\left[1 \ldots 2^{m}\right]$. Recall that more than $m$ bits are required to achieve a minimum Hamming distance $d_{\mathrm{f}}$ of at least two, as described in Section II. Assume that the interleaver $\pi$ in Fig. 1(c) places the $n_{\mathbf{t}}$ bits of the termination sequence $\mathbf{t}$ into the positions $\{\pi[j]\}_{j=1}^{n_{\mathrm{t}}}$ within the bit sequence $\mathbf{c}$. The set of corresponding impulse response final states can be represented using the $\left(n_{\mathbf{t}} \times m\right)$-element matrix $\mathbf{S}=\left[\begin{array}{llll}\mathbf{s}_{\pi[1]} & \mathbf{s}_{\pi[2]} & \ldots & \mathbf{s}_{\pi\left[n_{\mathbf{t}}\right.}\end{array}\right]^{\mathrm{T}}$. In the case where the bit sequence $\mathbf{b}^{\prime}$ in Fig. 1(c) contains only zero-valued bits, the set of final states that can be reached using the termination sequences $\mathbf{T}$ are represented by the rows of the $2^{m} \times m$ matrix $\mathbf{T} \cdot \mathbf{S}$, in accordance with (1).

If the rows of $\mathbf{T} \cdot \mathbf{S}$ represent every possible state in the set $\{0,1\}^{m}$, then the termination sequences $\mathbf{T}$ and the interleaver $\pi$ can terminate the inner encoder. Note that this is true, even if the bit sequence $\mathbf{b}^{\prime}$ in Fig. 1(c) does not contain zero-valued bits only, since the inner encoder has a linear response to these bits [21].

Let us now consider the design of termination sequences $\mathbf{T}$ for various binary recursive inner codes, as exemplified by the rate-1 code in Fig. 4. We consider codes having various numbers of memory elements $m \in\{1,2,3\}$ and various feedback 


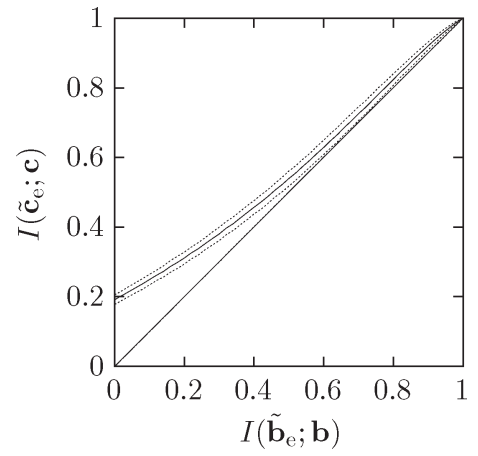

Fig. 3. EXIT band chart of the " $d_{f} \geq 2$ termination" scheme in Section II when transmitting frames comprising $n_{\mathbf{a}}=499$ source bits and $n_{\mathbf{d}}=999$ encoded bits over a BPSK-modulated uncorrelated narrow-band Rayleigh fading channel having an SNR of $E_{c} / N_{0}=0 \mathrm{~dB}$. Note that the corresponding EXIT band charts for the "no termination" and " $d_{f}=1$ termination" schemes are visually indistinguishable from this.

polynomials $\mathbf{f}$, as listed in Table I. Note that the bit sequences required to terminate a recursive inner code do not depend upon its generator polynomial $\mathrm{g}$. A full search was conducted to find termination sequences $\mathbf{T}$ that are optimal in the sense that they (in decreasing order of priority):

1) facilitate termination;

2) have a minimum Hamming distance of $d_{f} \geq 2$ between them;

3) have a minimal length $n_{\mathrm{t}}$;

4) have a maximal minimum Hamming distance $d_{\mathrm{f}}$ between them;

5) have a minimal fraction $a_{\mathrm{f}}$ of termination sequence pairs that are separated by the particular minimum maximal Hamming distance $d_{\mathrm{f}}$ achieved;

6) facilitate termination for a maximal fraction $r$ of the possible permutations of $\mathbf{S}$.

Note that owing to the periodicity of the impulse response final states, $r$ is also the fraction of all possible designs for the interleaver $\pi$ that facilitate termination. In all cases considered, we found that the set of optimal termination sequences $\mathbf{T}$ can be obtained as the set of codewords that can be generated by a particular linear block code, which may be described by a generator matrix $\mathbf{G}$. Table I provides the generator matrix $\mathbf{G}$ corresponding to each feedback polynomial $\mathbf{f}$ considered, together with the associated values of $d_{\mathrm{f}}, a_{\mathrm{f}}$, and $r$.

\section{EXIT CHART ANALYSIS}

Let us now consider our novel " $d_{\mathrm{f}} \geq 2$ termination" scheme's EXIT characteristics, as provided in Figs. 2 and 3. The transmission of uncoded bit sequences a having lengths of $n_{\mathbf{a}} \in\{4,49,499\}$ bits was considered. Note that an uncoded bit sequence length of $n_{\mathbf{a}}=4$ bits is so short that an optimal non-iterative full-search-based decoder would be preferred in practice. Despite this, however, it is useful for us to consider the iterative decoding of these short sequences here, since they highlight the issues considered in this paper.

The EXIT functions in Figs. 2 and 3 were obtained using an ( $R=1 / 2$ )-rate outer repetition code and the inner rate- 1 code of Fig. 4 in the scheme of Fig. 1(c). The rate-1 code employed

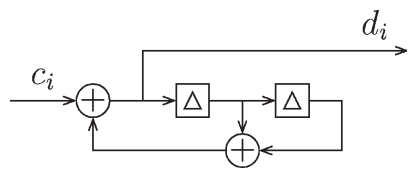

Fig. 4. Schematic of a binary recursive rate-1 convolutional code having $m=2$ memory elements, a feedback polynomial of $\mathbf{f}=\left[\begin{array}{lll}1 & 1 & 1\end{array}\right]$, and a generator polynomial of $\mathbf{g}=\left[\begin{array}{lll}1 & 0 & 0\end{array}\right]$.

has a feedback polynomial of $\mathbf{f}=\left[\begin{array}{lll}1 & 1 & 1\end{array}\right]$. This polynomial and the corresponding $\left(n_{\mathbf{t}}=3\right)$-bit terminator were selected from Table I, because they are associated with the lowest value of $r$. They therefore impose the highest grade restriction upon the design of the interleaver $\pi$, permitting us to assess the impact of this. As described in Section II, bit sequences $\mathbf{b}$ and $\mathbf{c}$ adopt lengths of $n_{\mathbf{b}}=n_{\mathbf{c}}=n_{\mathbf{a}} / R+n_{\mathbf{t}} \in\{11,101,1001\}$ bits for $n_{\mathbf{a}} \in\{4,49,499\}$, respectively. Note that the generator polynomial of the inner rate- 1 code in Fig. 4 is $g=\left[\begin{array}{lll}1 & 0 & 0\end{array}\right]$. As a result, the last $m$ number of bits output by the rate- 1 encoder will always equal the final state of its $m$ memory elements. Since this is known to the receiver when employing termination, there is no need to transmit these $m$ bits. The number of bits in the sequence $\mathbf{d}$ is therefore given by $n_{\mathbf{d}}=n_{\mathbf{a}} / R+n_{\mathbf{t}}-m \in$ $\{9,99,999\}$ for $n_{\mathbf{a}} \in\{4,49,499\}$, respectively. The transmission of these bits over a binary $(M=2)$ phase shift keying (BPSK)-modulated uncorrelated narrow-band Rayleigh fading channel having an SNR of $E_{c} / N_{0}=0 \mathrm{~dB}$ was simulated. Note that the corresponding EXIT charts in Figs. 2 and 3 are visually indistinguishable from those that may be obtained using an additive white Gaussian noise (AWGN) channel having an SNR of $E_{c} / N_{0}=-2 \mathrm{~dB}$.

Note that the EXIT functions shown in Figs. 2 and 3 are surrounded by EXIT bands [30]. These show the standard deviation that can be expected for the distribution of the iterative decoding trajectory corner points. As may be expected, the iterative decoding trajectories exhibit a greater variation from frame to frame when the frame length $n_{\mathbf{d}}$ is low. The simulations detailed in Section V revealed that the EXIT bands shown in Figs. 2 and 3 adequately reflected the variation exhibited by the iterative decoding trajectories, even in the case of $n_{\mathbf{d}}=9$. This may be attributed to our use of an $(R=1 / 2)$-rate outer repetition code, which obtains each pair of extrinsic LLRs in $\tilde{\mathbf{b}}_{\mathrm{e}}^{\prime}$ by simply swapping the corresponding pair of a priori LLRs in $\tilde{\mathbf{b}}_{\mathrm{a}}^{\prime}$. As a result, the $(R=1 / 2)$-rate outer repetition decoder does not assume the independence of its a priori LLRs, since only one of these contributes to each extrinsic LLR. For this reason, the deviation of the iterative decoding trajectories from the EXIT bands, owing to the dependence between the $a$ priori LLRs, was found to be significantly lower than what may typically be expected when employing short interleavers.

Note that Fig. 2 also provides EXIT characteristics for the "no termination" and the " $d_{\mathrm{f}}=1$ termination" schemes in Section II. To ensure fair comparisons, however, it is necessary for all schemes to generate encoded bit sequences $\mathbf{d}$ comprising the same number $n_{\mathbf{d}}$ of bits, when provided with the same input bit sequence a. For this reason, the "no termination" and the " $d_{\mathrm{f}}=1$ termination" schemes were emulated using the schematic of the " $d_{\mathrm{f}} \geq 2$ termination" scheme, which is 
provided in Fig. 1(c). More specifically, while the relevant generator matrix $\mathbf{G}$ in Table I yields the $\left(n_{\mathbf{t}}=3\right)$-bit termination sequences of $\mathbf{t} \in\left\{\left[\begin{array}{lll}0 & 0 & 0\end{array}\right],\left[\begin{array}{lll}0 & 1 & 1\end{array}\right],\left[\begin{array}{lll}1 & 0 & 1\end{array}\right],\left[\begin{array}{lll}1 & 1 & 0\end{array}\right]\right\}$ for the " $d_{\mathrm{f}} \geq 2$ termination" scheme, these sequences were exchanged for $\mathbf{t} \in$ $\left\{\left[\begin{array}{lll}0 & 0 & 0\end{array}\right],\left[\begin{array}{lll}0 & 0 & 1\end{array}\right],\left[\begin{array}{lll}0 & 1 & 0\end{array}\right],\left[\begin{array}{lll}0 & 1 & 1\end{array}\right]\right\}$ in the " $d_{\mathrm{f}}=1$ termination" scheme. Note that these replacement sequences are the $(m=$ 2 )-bit " $d_{\mathrm{f}}=1$ termination" sequences, concatenated onto a single zero-valued bit. The inclusion of this single bit represents doping [31], which is beneficial for iterative decoding. More specifically, since the receiver of the " $d_{\mathrm{f}}=1$ termination" scheme can expect the first bit in $\mathbf{t}$ to have a value of zero, it can always employ an infinite value for the first LLR in $\mathbf{t}_{\mathrm{e}}$, expressing absolute (and justified) confidence in a bit value of zero. As in the " $d_{\mathrm{f}} \geq 2$ termination" scheme, there is no need to transmit the last $m$ number of bits output by the rate1 encoder in the " $d_{\mathrm{f}}=1$ termination" scheme, since these will always equal its termination state. For this reason, in the " $d_{\mathrm{f}}=$ 1 termination" scheme, the number of bits in the sequence $\mathbf{d}$ is also given by $n_{\mathbf{d}}=n_{\mathbf{a}} / R+n_{\mathbf{t}}-m \in\{9,99,999\}$ for $n_{\mathbf{a}} \in\{4,49,499\}$, respectively.

By contrast, in the emulation of the "no termination" scheme, the bit sequence $\mathbf{t}=[0]$ was always employed. While this bit sequence does not facilitate termination at both ends of the inner decoder's trellis, it does provide $n_{\mathbf{t}}=1$ beneficial doping bit. Since the rate-1 encoder is not terminated in the "no termination" scheme, it is necessary to transmit all of the bits output by the rate- 1 encoder. Therefore, the employment of just $n_{\mathbf{t}}=1$ bit in the sequence $\mathbf{t}$ ensures that the number of transmitted bits is the same as in the " $d_{\mathrm{f}} \geq 2$ termination" and " $d_{\mathrm{f}}=1$ termination" schemes, namely, $n_{\mathbf{d}}=n_{\mathbf{a}} / R+n_{\mathbf{t}} \in$ $\{9,99,999\}$ for $n_{\mathbf{a}} \in\{4,49,499\}$, respectively.

Note that the only differences between the three schemes considered are the sets of possible values for $\mathbf{t}$ and the corresponding limitations that are placed upon the design of the interleaver $\pi .^{2}$ Since these limitations are strictest for our proposed " $d_{\mathrm{f}} \geq 2$ termination" scheme, we consider our comparisons to be unbiased. Note that, while the complexity associated with termination differs in the three schemes considered, we can expect these differences to be negligible for frame lengths of $n_{\mathbf{d}}=99$ and $n_{\mathbf{d}}=999$ bits, since termination affects only $n_{\mathbf{t}}=3$ of these bits. From this perspective, it can be said that termination is associated with only a negligible complexity cost.

Observe in Fig. 2 that the terminated recursive inner codes employed in the " $d_{\mathrm{f}}=1$ termination" and " $d_{\mathrm{f}} \geq 2$ termination" schemes have EXIT functions that reach the

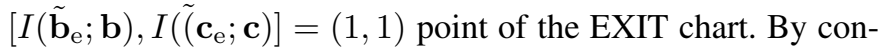
trast, the unterminated inner code of the "no termination"

\footnotetext{
${ }^{2}$ When employing a rate-1 inner encoder having a generator polynomial of the form $\mathbf{g}=[1,0,0, \ldots, 0]$, it is in fact necessary to interleave the termination sequence $\mathbf{t}$ of the " $d_{\mathrm{f}}=2$ termination" scheme to distinguish it from the "no termination" scheme. If the $m$ termination bits of $\mathbf{t}$ were merely appended to the end of the rate-1 encoder's input bit sequence, like in Fig. 1(b), then they would only influence the value of the last $m$ bits output by the encoder. Since these bits are not transmitted when employing termination, the bits that are transmitted $\mathbf{d}$ would only depend upon the source bit sequence $\mathbf{a}$, like in the "no termination" scheme. For this reason, the " $d_{\mathrm{f}}=2$ termination" scheme becomes equivalent to the "no termination" scheme, unless its termination sequence is interleaved, like in Fig. 1(c).
}

scheme has an EXIT function that does not reach the $(1,1)$ point, as predicted in Section II. The shortcomings of the "no termination" scheme are demonstrated most clearly in Fig. 2(a), which considers the case of very short coded frames comprising just $n_{\mathbf{d}}=9$ bits. Here, the inner decoder provides extrinsic LLRs $\tilde{\mathbf{c}}_{\mathrm{e}}$ having a mutual information of just $I\left(\tilde{\mathbf{c}}_{\mathrm{e}} ; \mathbf{c}\right)=0.771$ when provided with a priori LLRs $\tilde{\mathbf{c}}_{\mathrm{a}}$ having a mutual information of $I\left(\tilde{\mathbf{c}}_{a} ; \mathbf{c}\right)=1$. As shown in Figs. 2(d) and 3, however, this problem is mitigated (although not eliminated) as the frame length is increased. Indeed, the "no termination" scheme's inner decoder is capable of providing an extrinsic information $\tilde{\mathbf{c}}_{\mathrm{e}}$ having mutual informations of up to $I\left(\tilde{\mathbf{c}}_{\mathrm{e}}\right)=0.979$ and $I\left(\tilde{\mathbf{c}}_{\mathrm{e}}\right)=0.999$ for $n_{\mathbf{d}}=99$ and $n_{\mathbf{d}}=999$, respectively.

Let us now turn our attention to the outer EXIT functions provided in Figs. 2 and 3. Similar to the EXIT functions of irregular codes [8], these are obtained as a weighted average of the EXIT functions belonging to the half-rate repetition code and terminator in Fig. 1(c). Here, a weight of $\left(n_{\mathbf{b}}-n_{\mathbf{t}}\right) / n_{\mathbf{b}}$ is applied to the half-rate repetition code's EXIT function, since it provides $\left(n_{\mathbf{b}}-n_{\mathbf{t}}\right)$ of the $n_{\mathbf{b}}$ bits in $\mathbf{b}$. Meanwhile, the EXIT function of the terminator is associated with a weight of $n_{\mathbf{t}} / n_{\mathbf{b}}$, which diminishes as $n_{\mathbf{b}}$ (and, hence, $n_{\mathbf{d}}$ ) is increased. It is for this reason that the outer EXIT functions in Figs. 2 and 3 resemble the diagonal EXIT function of the half-rate repetition code to an increasingly greater degree as $n_{\mathbf{d}}$ is increased.

Observe in Fig. 2 that the "no termination" and " $d_{\mathrm{f}} \geq 2$ termination" schemes have inverted outer EXIT functions that reach the $\left[I\left(\tilde{\mathbf{b}}_{\mathrm{e}} ; \mathbf{b}\right), I\left(\tilde{\mathbf{c}}_{\mathrm{e}} ; \mathbf{c}\right)\right]=(1,1)$ point of the EXIT chart. By contrast, the inverted outer EXIT function of the " $d_{\mathrm{f}}=1$ termination" scheme has an EXIT function that does not reach the $(1,1)$ point in this way. This may be attributed to the unavailability of any extrinsic information pertaining to the two terminating bits of $\mathbf{t}$ that are employed in the " $d_{\mathrm{f}}=1$ termination" scheme. As a result, the corresponding LLRs in $\mathbf{t}_{\mathrm{e}}$ must be allocated zero-valued LLRs, as described in Section II. This yields an extrinsic mutual information $I\left(\tilde{\mathbf{b}}_{\mathrm{e}} ; \mathbf{b}\right)$ of less than unity, even when the a priori mutual information $I\left(\tilde{\mathbf{b}}_{\mathrm{a}} ; \mathbf{b}\right)$ is unity. Indeed, the " $d_{\mathrm{f}}=1$ termination" scheme is only capable of providing extrinsic mutual informations of up to $I\left(\tilde{\mathbf{b}}_{\mathrm{e}} ; \mathbf{b}\right)=0.818, I\left(\tilde{\mathbf{b}}_{\mathrm{e}} ; \mathbf{b}\right)=0.980$, and $I\left(\tilde{\mathbf{b}}_{\mathrm{e}} ; \mathbf{b}\right)=0.998$ for $n_{\mathbf{d}}=9, n_{\mathbf{d}}=99$, and $n_{\mathbf{d}}=999$, respectively.

As highlighted in Fig. 2(a)-(c), our novel " $d_{\mathrm{f}} \geq 2$ termination" scheme is the only one that can create an open EXIT chart tunnel to the $(1,1)$ point.

Note that, in the "no termination" scheme, the "terminator" block in the receiver of Fig. 1(c) has an inverted EXIT function that resembles a vertical line at $I\left(\tilde{\mathbf{b}}_{\mathrm{e}} ; \mathbf{b}\right)=1$, since it always provides an infinite value for the $n_{\mathbf{t}}=1$ extrinsic LLR of $\tilde{\mathbf{t}}_{\mathrm{e}}$. Similarly, the terminator of the " $d_{\mathrm{f}}=1$ termination" scheme has an inverted EXIT function that resembles a vertical line at $I\left(\tilde{\mathbf{b}}_{\mathrm{e}} ; \mathbf{b}\right)=0.333$, since it provides an infinite value for one of the $n_{\mathbf{t}}=3$ extrinsic LLRs in $\mathbf{t}_{\mathrm{e}}$. It is for this reason that the inverted outer EXIT functions of the "no termination" and " $d_{\mathrm{f}}=1$ termination" schemes commence at points $I\left(\tilde{\mathbf{b}}_{\mathrm{e}} ; \mathbf{b}\right)>$ 0 along the horizontal axis of the EXIT charts. This is in contrast to the " $d_{\mathrm{f}} \geq 2$ termination" scheme, which is unable to generate any extrinsic information $\tilde{\mathbf{b}}_{\mathrm{e}}$ in the absence of any a priori information $\tilde{\mathbf{b}}_{\mathrm{a}}$, since it does not employ doping. 
Observe that the area beneath the outer EXIT function provided in Fig. 2(a) for the "no termination" scheme is approximately equal to the overall coding rate of $n_{\mathbf{a}} / n_{\mathbf{d}}=0.444$ [32]. By contrast, the terminated schemes have corresponding areas in Fig. 2(b) and (c) of about $\left(n_{\mathbf{a}}+m\right) / n_{\mathbf{b}}=0.545$. In all cases, the areas beneath the inner EXIT functions become equal to those of the outer EXIT functions at an SNR of $-2.18 \mathrm{~dB}$ for the Rayleigh fading channel and $-3.58 \mathrm{~dB}$ for the AWGN channel. Note that these are the SNRs at which the channel capacity equals the throughput $n_{\mathbf{a}} / n_{\mathbf{d}} \cdot \log _{2}(M)=0.444$ of the schemes, indicating that none of them suffer from rate loss [32].

\section{Bit-Error-Rate Performance}

In this section we consider the BER performance that can be achieved by the "no termination," " $d_{\mathrm{f}}=1$ termination," and " $d_{\mathrm{f}} \geq 2$ termination" schemes in Section IV when employing various frame lengths. This was investigated by simulating the transmission of randomly generated frames over BPSKmodulated uncorrelated narrow-band Rayleigh fading channels and AWGN channels having various SNRs per bit $E_{b} / N_{0}=$ $n_{\mathbf{d}} / n_{\mathbf{a}} \cdot E_{c} / N_{0}$. In each case, the number of frames considered was adjusted to ensure that a statistically significant number of bit errors was observed. A different random design was employed for the interleaver $\pi$ in each frame, since this is assumed by the EXIT band chart analysis in Section IV [30]. Note that, in the case of the terminated schemes, the resultant interleaver designs were rejected if they would not facilitate termination, as detailed in Section III.

In all simulations, iterative decoding was continued until convergence was detected. As may be expected, fewer iterations were required to achieve this when the $E_{b} / N_{0}$ value was high. At a Rayleigh fading channel $E_{b} / N_{0}$ value of $10 \mathrm{~dB}$ and an AWGN channel $E_{b} / N_{0}$ value of $4 \mathrm{~dB}$, we found that the schemes employing frame lengths of $n_{\mathbf{d}}=9, n_{\mathbf{d}}=99$, and $n_{\mathbf{d}}=999$ typically required four, five, and six iterations to achieve convergence, respectively. Our BER results are presented in Figs. 5 and 6.

Observe in Figs. 5 and 6 that improved BERs are offered when longer frames are employed. This behavior is typical of iteratively decoded schemes, since higher frame lengths permit the iterative decoding trajectories to more readily navigate through the EXIT chart tunnel [1]. In all cases, the " $d_{\mathrm{f}} \geq 2$ termination" scheme can be seen to offer the most desirable performance at high $E_{b} / N_{0}$ values. This may partially be attributed to its EXIT functions, which do not intersect each other before the $(1,1)$ point of the EXIT chart, like those of the "no termination" and " $d_{\mathrm{f}}=1$ termination" schemes did, as described in Section IV. Furthermore, owing to the increased Hamming distance of $d_{\mathrm{f}}=2$ between the various termination sequences of the " $d_{\mathrm{f}} \geq 2$ termination" scheme, it was found to offer better distance properties and, hence, lower error floors than the "no termination" and " $d_{\mathrm{f}}=1$ termination" schemes.

More specifically, owing to the selection of outer and inner codes employed, the minimum Hamming distance between any two legitimate permutations of the bit sequence $\mathbf{d}$ was found

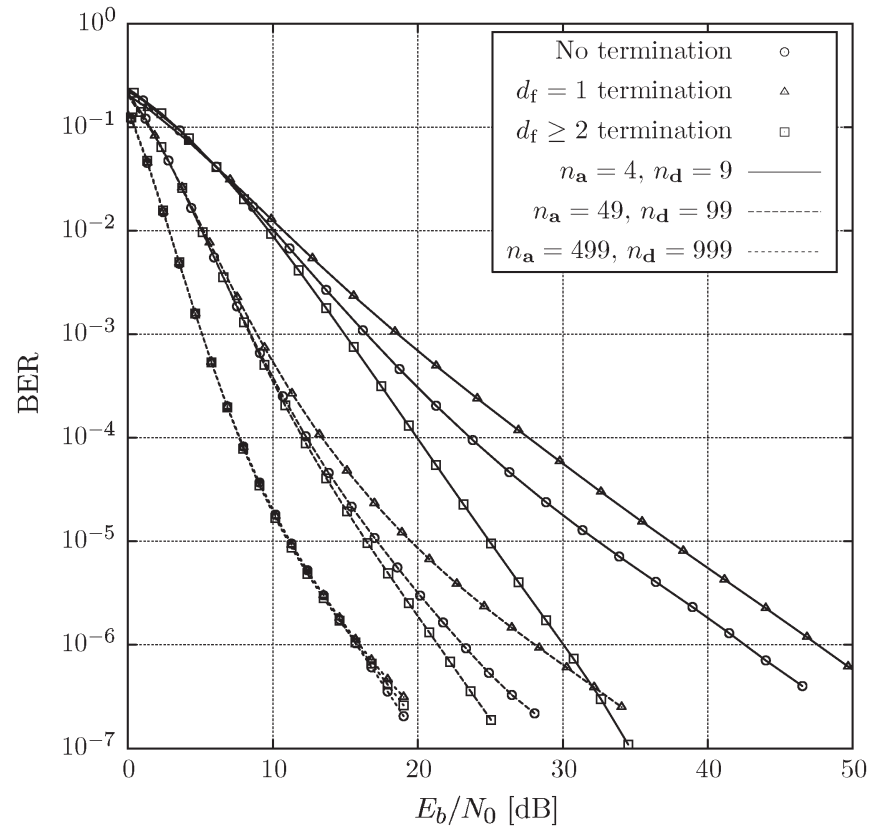

Fig. 5. BER versus $E_{b} / N_{0}$ performance of the schemes introduced in Section II when transmitting frames having various lengths over a BPSKmodulated uncorrelated narrow-band Rayleigh fading channel.

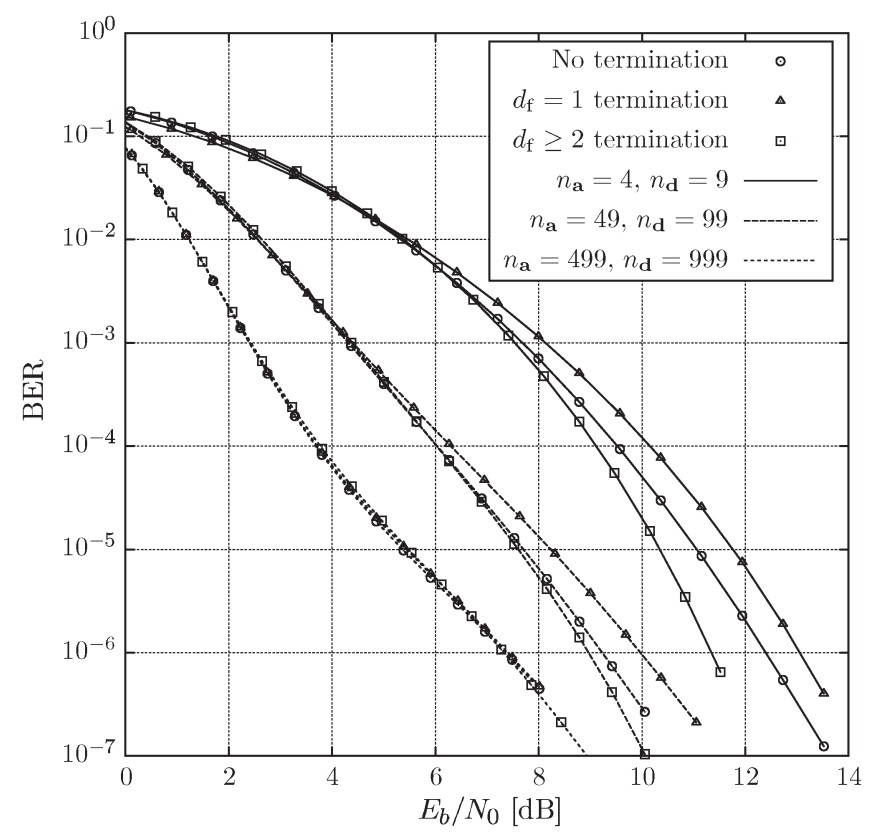

Fig. 6. BER versus $E_{b} / N_{0}$ performance of the schemes introduced in Section II when transmitting frames having various lengths over a BPSKmodulated AWGN channel.

to be two in the " $d_{\mathrm{f}} \geq 2$ termination" scheme, irrespective of the interleaver design. The "no termination" scheme was also found to have a minimum Hamming distance of two but with higher multiplicities, explaining the higher error floors shown in Figs. 5 and 6. Note that the " $d_{\mathrm{f}}=1$ termination" scheme reduces the minimum Hamming distance to one whenever its interleaver adopts an unfortunate design, causing its error floors to be the highest of all. This is surprising, since termination typically improves the distance properties of turbo codes [21]. 
We shall explore this finding in a future publication, where we shall determine the conditions in which the " $d_{\mathrm{f}}=1$ termination" scheme degrades the minimum Hamming distance.

For a frame length of $n_{\mathbf{d}}=999$ bits, the three schemes can be seen to offer similar BER performance in Figs. 5 and 6. This may be explained by the shifting of the intersection point of the "no termination" and " $d_{\mathrm{f}}=1$ termination" schemes' EXIT functions toward the $(1,1)$ point, as described in Section IV. Furthermore, it was found that the conditions required for the " $d_{\mathrm{f}}=1$ termination" scheme to adopt a minimum Hamming distance of one are more rarely satisfied as its frame length increases.

\section{CONCLUSION}

In this paper, we have proposed a novel method for terminating recursive inner codes in serial concatenations. Our method employs termination sequences having a minimum Hamming distance of at least two between them, as described in Section II. In Section IV, we have demonstrated that our approach facilitates the creation of an open EXIT chart tunnel to the $(1,1)$ point of the EXIT chart, where the ML BER performance is obtained. By contrast, when termination is omitted or the " $d_{\mathrm{f}}=1$ termination" method in [6], [9]-[11], [19], and [20] is employed, the EXIT functions intersect each other before the $(1,1)$ point, as shown in Section IV. As a result, our novel approach facilitates useful BER reductions when employing transmission frames that are on the order of 100 bits long, which are typical in wireless sensor networks, for example. Indeed, Fig. 5 shows that our proposed " $d_{\mathrm{f}} \geq 2$ termination" scheme can achieve a BER of $10^{-6}$ at an $E_{b} / N_{0}$ value that is $6.5 \mathrm{~dB}$ lower than that required by a " $d_{\mathrm{f}}=1$ termination" benchmarker when employing an encoded frame length of $n_{\mathbf{d}}=99$ bits and transmitting over an uncorrelated Rayleigh fading channel. In the case of an AWGN channel, a corresponding gain of $1 \mathrm{~dB}$ was obtained, as shown in Fig. 6 . These results are particularly remarkable, because in this comparison, the termination method employed affected the values of only $n_{\mathbf{t}}=3$ of the $n_{\mathbf{b}}=101$ bits that were encoded by the inner encoder. Furthermore, these gains were obtained without incurring a significantly higher computational complexity, as described in Section IV.

\section{REFERENCES}

[1] S. ten Brink, "Convergence of iterative decoding," Electron. Lett., vol. 35, no. 10, pp. 806-808, May 1999.

[2] S. Benedetto and G. Montorsi, "Serial concatenation of block and convolutional codes," Electron. Lett., vol. 32, no. 10, pp. 887-888, May 1996.

[3] S. Benedetto and G. Montorsi, "Iterative decoding of serially concatenated convolutional codes," Electron. Lett., vol. 32, no. 13, pp. 1186-1188, Jun. 1996.

[4] C. Berrou, A. Glavieux, and P. Thitimajshima, "Near Shannon limit errorcorrecting coding and decoding: Turbo-codes. 1," in Proc. IEEE Int. Conf. Commun., Geneva, Switzerland, May 1993, vol. 2, pp. 1064-1070.

[5] D. Divsalar, H. Jin, and R. J. McEliece, "Coding theorems for 'turbo-like' codes," in Proc. Allerton Conf. Commun., Control Comput., Urbana, IL, Sep. 1998, pp. 201-210.

[6] R. Garello, P. Pierleoni, and S. Benedetto, "Computing the free distance of turbo codes and serially concatenated codes with interleavers: Algorithms and applications," IEEE J. Sel. Areas Commun., vol. 19, no. 5, pp. 800812, May 2001
[7] D. Divsalar, S. Dolinar, and F. Pollara, "Serial concatenated trellis coded modulation with rate-1 inner code," in Proc. IEEE Global Telecommun. Conf., San Francisco, CA, Nov. 2000, vol. 2, pp. 777-782.

[8] M. Tüchler and J. Hagenauer, "EXIT charts of irregular codes," in Proc. Conf. Inf. Sci. Syst., Princeton, NJ, Mar. 2002, pp. 748-753.

[9] I. Altunbas and A. Yongacoglu, "Performance of serially concatenated space-time QPSK systems," in Proc. IEEE Int. Symp. Comput. Commun., Antalya, Turkey, Jun. 2003, pp. 271-276.

[10] S. X. Ng, J. Y. Chung, and L. Hanzo, "Turbo-detected unequal protection MPEG-4 wireless video telephony using multilevel coding, trellis coded modulation and space-time trellis coding," Proc. Inst. Elect. Eng.-Commun., vol. 152, no. 6, pp. 1116-1124, Dec. 2005.

[11] I. Altunbas, "Performance of serially concatenated coding schemes for MIMO systems," Int. J. Electron. Commun., vol. 61, no. 1, pp. 1-9, Jan. 2007.

[12] R. G. Maunder, J. Kliewer, S. X. Ng, J. Wang, L.-L. Yang, and L. Hanzo, "Joint iterative decoding of trellis-based VQ and TCM," IEEE Trans. Wireless Commun., vol. 6, no. 4, pp. 1327-1336, Apr. 2007.

[13] R. G. Maunder, J. Wang, S. X. Ng, L.-L. Yang, and L. Hanzo, "On the performance and complexity of irregular variable length codes for near-capacity joint source and channel coding," IEEE Trans. Wireless Commun., vol. 7, no. 4, pp. 1338-1347, Apr. 2008.

[14] R. G. Maunder and L. Hanzo, "Genetic algorithm aided design of component codes for irregular variable length coding," IEEE Trans. Commun., vol. 57, no. 5, pp. 1290-1297, May 2009.

[15] R. G. Maunder and L. Hanzo, "Near-capacity irregular variable length coding and irregular unity rate coding," IEEE Trans. Wireless Commun., to be published.

[16] M. Tüchler, "Design of serially concatenated systems depending on the block length," IEEE Trans. Commun., vol. 52, no. 2, pp. 209-218, Feb. 2004.

[17] J. Kliewer, A. Huebner, and D. J. Costello, "On the achievable extrinsic information of inner decoders in serial concatenation," in Proc. IEEE Int. Symp. Inf. Theory, Seattle, WA, Jul. 2006, pp. 2680-2684.

[18] J. Kliewer, N. Görtz, and A. Mertins, "Iterative source-channel decoding with Markov random field source models," IEEE Trans. Signal Process. vol. 54, no. 10, pp. 3688-3701, Oct. 2006.

[19] J. Freudenberger, R. Jordan, M. Bossert, and S. Shavgulidze, "Serially concatenated convolutional codes with product distance," in Proc. Int. Symp. Turbo Codes, Brest, France, Sep. 2000, pp. 81-84.

[20] Y. Yao and M. M. K. Howlader, "Serial concatenated single differential space-time coded OFDM system," in Proc. IEEE Int. Conf. Commun., Anchorage, AK, May 2003, vol. 5, pp. 3150-3154.

[21] O. Joerssen and H. Meyr, "Terminating the trellis of turbo-codes," Electron. Lett., vol. 30, no. 16, pp. 1285-1286, Aug. 1994.

[22] A. S. Barbulescu and S. S. Pietrobon, "Terminating the trellis of turbocodes in the same state," Electron. Lett., vol. 31, no. 1, pp. 22-23, Jan. 1995.

[23] W. J. Blackert, E. K. Hall, and S. G. Wilson, "Turbo code termination and interleaver conditions," Electron. Lett., vol. 31, no. 24, pp. 2082-2084, Nov. 1995

[24] M. C. Reed and S. S. Pietrobon, "Turbo-code termination schemes and a novel alternative for short frames," in Proc. IEEE Int. Symp. Pers., Indoor, Mobile Radio Commun., Taipei, Taiwan, Oct. 1996, vol. 2, pp. 354-358.

[25] J. Hokfelt, O. Edfors, and T. Maseng, "A survey on trellis termination alternatives for turbo codes," in Proc. IEEE Veh. Technol. Conf., Houston, TX, Jul. 1999, vol. 3, pp. 2225-2229.

[26] P. Elias, "Coding for noisy channels," IRE Int. Conf. Rec., vol. 3, no. 4, pp. 37-46, 1955 .

[27] G. D. Forney, "The Viterbi algorithm," Proc. IEEE, vol. 61, no. 3 , pp. 268-278, Mar. 1973.

[28] J. B. Anderson and S. M. Hladik, "Tailbiting MAP decoders," IEEE J. Sel. Areas Commun., vol. 16, no. 2, pp. 297-302, Feb. 1998.

[29] L. Bahl, J. Cocke, F. Jelinek, and J. Raviv, "Optimal decoding of linear codes for minimizing symbol error rate (Corresp.)," IEEE Trans. Inf. Theory, vol. IT-20, no. 2, pp. 284-287, Mar. 1974.

[30] J. W. Lee and R. E. Blahut, "Generalized EXIT chart and BER analysis of finite-length turbo codes," in Proc. IEEE Global Telecommun. Conf., San Fransisco, CA, Dec. 2003, vol. 4, pp. 2067-2072.

[31] S. ten Brink, "Code doping for triggering iterative decoding convergence," in Proc. IEEE Int. Symp. Inf. Theory, Washington, DC, Jun. 2001, p. 235.

[32] A. Ashikhmin, G. Kramer, and S. ten Brink, "Code rate and the area under extrinsic information transfer curves," in Proc. IEEE Int. Symp. Inf. Theory, Lausanne, Switzerland, Jun. 2002, p. 115. 


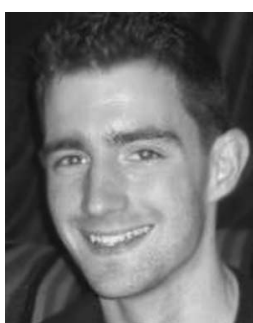

Robert G. Maunder (M'03) received the B.Eng. (first-class honors) degree in electronic engineering and the Ph.D. degree in wireless communications from the University of Southampton, Hampshire, U.K., in July 2003 and December 2007, respectively. Since December 2007, he has been a Lecturer with the School of Electronics and Computer Science, University of Southampton. His research interests include joint source/channel coding, iterative decoding, irregular coding, and modulation techniques. He has published a number of IEEE papers in these

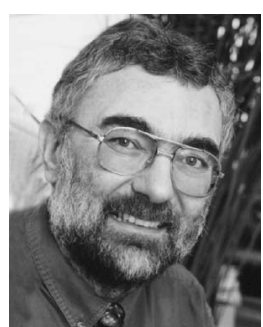

Lajos Hanzo (F'04) received the Dipl.Ing. (Master's) and Ph.D. degrees from the Technical University of Budapest, Budapest, Hungary, in 1976 and 1983, respectively, and the D.Sc. degree from the University of Southampton, Hampshire, U.K., in 2004.

During his career in telecommunications, he has held various research and academic posts in Hungary, Germany, and the U.K. Since 1986, he has been with the School of Electronics and Computer Science, University of Southampton, where he holds the Chair in telecommunications. He is an enthusiastic supporter of industrial and academic liaison, and he offers a range of industrial courses. Currently, he heads an academic research team, working on a range of research projects in the field of wireless multimedia communications sponsored by the industry, the Engineering and Physical Sciences Research Council, U.K., the European IST Program, and the Mobile Virtual Centre of Excellence, U.K. He is the author or a coauthor of 17 books, totaling 10000 pages on mobile radio communications, and about 900 research papers.

Dr. Hanzo is a Fellow of the Royal Academy of Engineering and the Institute of Engineering of Technology (IET). He is an IEEE Distinguished Lecturer. He is the Editor-in-Chief of the IEEE Press and an editorial board member of the PROCEEDINGS OF THE IEEE, as well as a Governer of both the IEEE Vehicular Technology and IEEE Communications Societies. He has acted as the Technical Program Committee Chair of numerous major IET and IEEE conferences, has presented various keynote lectures, and has received a number of distinctions. 